\title{
CITY CLEANLINESS DRIVE WEB PORTAL USING REGION BASED CONVOLUTIONAL NEURAL NETWORKS
}

\author{
Akila V ${ }^{1}$, Kasi Glory ${ }^{1}$, Pinnamaraju Sanjana Varma ${ }^{1}$, Puchakayala Lakshmi Hemanjili ${ }^{1}$,Tutari Vijaya Lohitha ${ }^{1}$ and T.Sheela ${ }^{2}$
}

${ }^{1}$ Gokaraju Rangaraju Institute of Engineering and Technology, Hyderabad

${ }^{2}$ Sri Sai Ram Engineering College, Chennai

\begin{abstract}
Identification or detection of object played an important role in Computer Vision, in implementations like city construction process Managers had often wasted lot of their energy, time and resources in cleaning up the garbage, which was unexpectedly showed up. When deep network systems increased its complexity, the systems are constrained by the training data availability. Due to this, Open $\mathrm{CV}$, Google AI released the Open images dataset publicly, so that the research and development would happen in study and analysis of images. As a result, virtual street cleanliness been at most important in this project, however the existing system has disadvantages like collection of garbage is not automated. It doesn't use the best real time algorithm for identifying the objects. This project will embed the above said things in the system, making the work of managers to keep the city/construction site clean very simple and effective.
\end{abstract}

Keywords: Computer Vision, Edge Computing, Neural Network

\section{Introduction}

Keeping our locality clean is very important for every one of us, it involves tasks such as sweeping, litter picking, removal of disturbing graffiti, when these tasks are done poorly or ineffectively the outcome of this would be clearly visible causing impact on the quality of our lives, and in extreme cases it could be shelter for beings like mosquitoes, which cause severe diseases like malaria, dengue questioning the health of the people living in the locality. And it also said by some that there are links between the environmental problems and crimes in the city [1]. But on the other hands if the system is effective in cleaning up the locality, people's life style would be better, which can help urban development, it makes the locality to look beautiful and attractive giving us a mental and physical advantages and also it attracts tourists which could make income [2].

The ground water which is a drinking source for many will be pure and portable. And hence many researches had been taken in this field of garbage detection causing many automated methods to come into existence, using a camera installed in moving vehicles which clicks real time locality photos, The photos are analyzed and the garbage is detected by the system using different algorithms, once detected the report would be sent to city managers, which makes their work easy to clean the localities [3].

As the above said information been the background of our project, The objectives of our project is to create a website that keeps track of garbage complaints, then to classify the level of garbage at the locality as very high, high, medium, low using garbage count and then send the analyzed report to the city managers, this project integrates the machine learning technology with the web portal, and creates a fully functional web portal for garbage complaints [4].

Many researches, developments had been taken place in the field of object detection, the existing system could detect the presence of the garbage, and however it couldn't specify the exact boundaries of the garbage. System had introduced a novel framework for edge computing.

There is a presence of edge layer between the terminals and the cloud server. Edge server helps to manage a portion of resources at the edge layer, it also helps to store data temporarily and convey data resources in time, the disadvantages of this system include the requirement of huge dataset causing the training time to. be significant [5].

In this proposed system object localization was used to detect the bounding boxes that is the exact location of the garbage. It also classifies the garbage into high, medium, low levels based on the garbage count, incorporated graphical analysis to view the garbage as count. They have a self-collected public garbage data set, which has been used as a benchmark for street cleanliness evaluation and garbage detection and the density of the garbage in the image. The web portal which was designed is scalable and portable. In addition to that the data collected is used to have a map of visualstreet cleaning [6]. 


\section{Region Based Convolutional Neural Networks(R-CNN)}

The flow chart shows the flow of the program that works within the model. The R-CNN deep learning model will first take the images and passes it to the neural network [7]. The neural network will calculate the garbage in the image using object detection and followed by canny edge detection which will give the count of the total garbage present in the place then it adds the information to the total garbage present in that area and then compares if the total garbage count present in the area with the defined criteria of low, medium and high garbage areas that accordingly the result is noted for the analysis and to create the analysis view to the admin and the user.

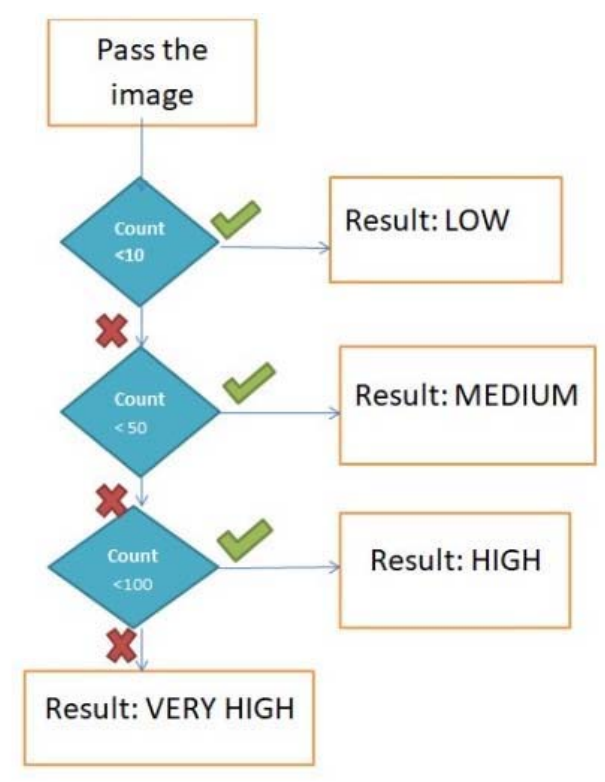

Fig.1. Shows the flow of Web Portal

Here it will mainly discuss the development details while fulfilling the project requirements. First, it will look implementation tools - which are required for creating the web application and Machine Learning model development, describing both the server-side and client-side implementation of the system. The serverside implementation will be mainly consisting of architecture layers and the algorithm of data processing that is the RCNN. Client-side development is concerned with building the user interface for the application. Then the data (Images) collection, The deep learning algorithm with the description of the approach and finally, multi-level garbage assessment of the approach [8]. It has used many on-going technologies in order to deploy the project successfully. Web Applications involve Django for design and frame work.
Registration is available for the user. Any citizen of the city can register with the portal using this registration page and this registered user needs to provide with some details such as his name, phone number, email id, age, address, pin code [9]. All the details given by user will be stored so that admin can have the details of the registered user and count on his reports. This information will be helpful to find the responsibility showed by any user. The registered user can report the garbage found in the surrounding area to the administrator and the one who is in charge of cleaning the garbage. The user will have to take certain pictures and report the garbage. The user has to give some additional details such as the location of the area where the garbage is present, including the address and the landmark. They can also define the subject of the garbage. After the report is given, they can also followup with the problem by giving the feedback and the feedback page consists of details about the previously reported cases and also the ratings. They can be the key to the admin for taking better action that satisfies the people and the government. This feature helps the user to give valuable feedback that could help the administration to work hard to give the best result. It contains the information about the user and the feedback.

Garbage Count detection and counting as shown in Fig.2. The admin will be able to see the garbage and count of the garbage at a place and they will be getting the details of the amount of garbage present in certain area and all the reports for a single place is given to the admin to react according to the level of the garbage present. This database that contains the count of the garbage is stored from the values generated by the algorithm such as id, user id and garbage count. Another module is the send query where the admin can also send query to the respective user. If there exists any query regarding the garbage and they can also take action on this by sending the information to the supervisor in charge of cleaning. This table consists of the details that are stored when the admin send the query, the attributes are the id, report number and the query details along with the name.

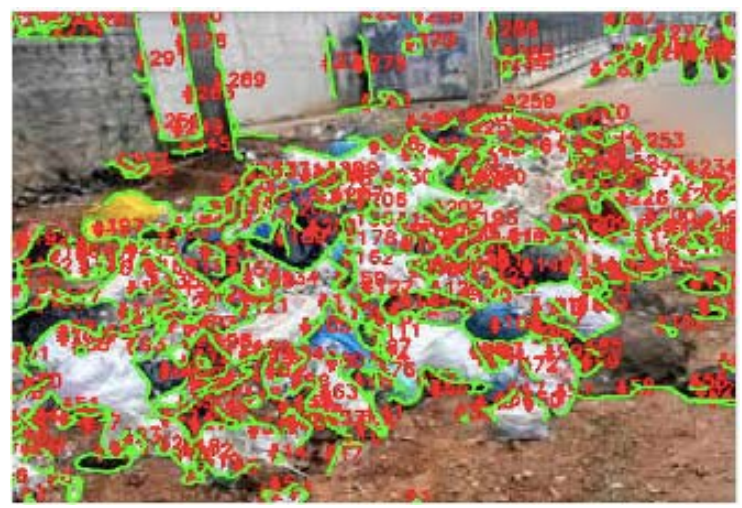

Fig.2. Image shows the detection and counting of garbage in the picture 
Edge computing will use less resource when compared to other cloud computing techniques; the difference between them is that some services will be processed on the edge before itself when a huge data is generated. PyCharm is used to develop this project integrate, Django with python to build the application and Deep learning is used for image detection and computing[10].

$\mathrm{R}-\mathrm{CNN}$ is the algorithm that is one of the most used algorithms used in image recognition in the field of Deep Learning. In the given project, we have created a street cleanliness assessment approach. And the approach combines mobile edge computing and Region Based Convolutional neural network to identify the garbage. This approach is mainly composed of three parts. They are Data Classification, Data Preprocessing and R-CNN Model

\section{Data Classification}

The first step is acquiring the data and using it for classification. In real time, the city administrators or the one who is in charge of this particular work will have the control through mobile device for collecting the image data and take actions against the reports based on the level of cleanliness given by the algorithm[11]. There are some points to be noted before taking the pictures. The first thing is that the distance between the picturing points. The distance at which the garbage is shot is to be taken into consideration the vehicle that is used to clean the garbage will be taking the pictures according to the rules that are set by the administrator. Basically, taking the shoot range as 150 to 300 meters far.

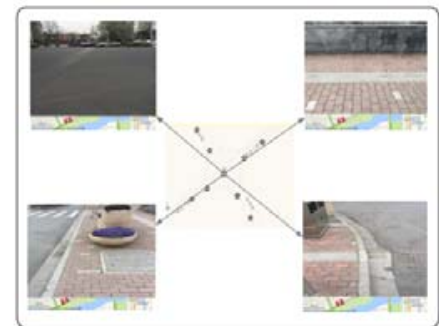

Fig 3. Shows the multiple pictures of same area[5]

The standard image resolution of shooting points using mobile stations as shown in Fig.3. by taking multiple pictures at the same place from different directions and each with shooting point.

\section{Data Pre-processing}

In this step, the system will be using edge servers to for storing the image that is captured and all other data related to the image in the mobile station and proceed with the image pre-processing techniques that are provided in the model developed.

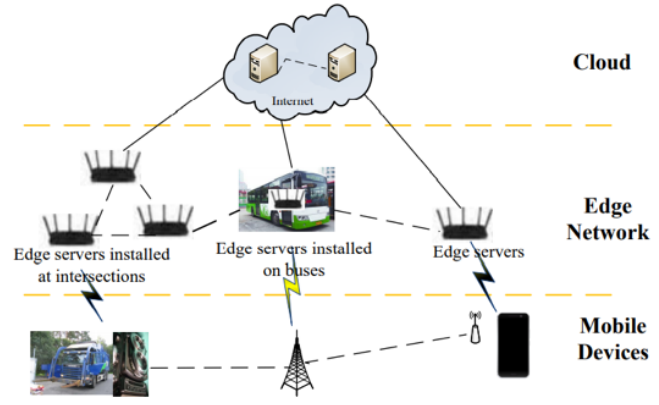

Fig.4. Network Connections[5]

Then, the edge server will eliminate the images that do not contain any kind of garbage and the images that has road areas and then use techniques such as the linear normalization to change all the images to common numbered pixel-images and these processed images canny edge detection is applied and are then sent to the cloud for waste material detection as shown in Fig.4.

\section{R-CNN Model and Cleanliness Calculation}

Servers are used to do multiple things. The firstly it is used for improving the ability to work of the entire system. At the first stage, the garbage detected part of the data from the image is collected and given as the input to the R-CNN network and then other parameters of the image is taken care such as the size of the image, size of all the images are modified to the standard defined size.

The model that builds consists of an algorithm that modifies the size of the Image automatically as soon as the image is uploaded. The second task is to differentiate between the informative and noninformative data to filter the informative from the noninformative data. For example, it might come across some problematic pictures that include useless data such as the trees, vehicles moving around and children playing etc. For the whole garbage detection system, pictures which do not have full street images are not useful in any way. In order to make good use of the resources that are images and also reduce the wastage of time during the next steps as shown in Fig.5.

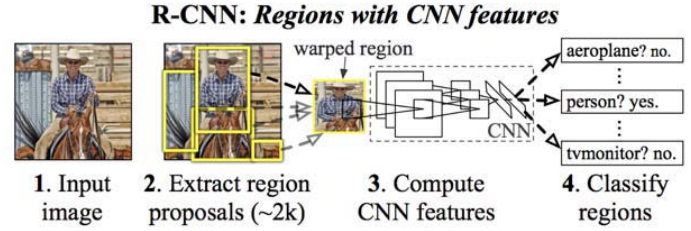

Fig 5. RCNN model form Image Classification[6]

Then a model is trained by selecting necessary prerequisites and epochs to detect garbage on the street. In this stage the algorithm works on the data points and 
images that it learned previously at the training phase and figures out the amount of data present in each picture here the program used a count variable to calculate the amount of garbage present in that particular picture. Finally, depending on the results of the above procedure, city cleanliness level is calculated and is divided into different levels.

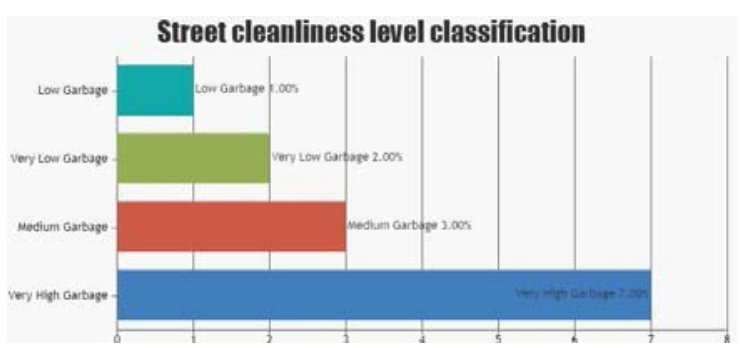

Fig 6. Analysis of the report

Fig.6 shows analysis of the cleanliness level by classifying then into different categories based on the level of cleanliness. It is classified as very high, high, medium, low using garbage count and then send the report to managers.

\section{CONCLUSIONS}

Day by day development in the modern technologies has encouraged many cities to become into smart cities. And now street cleanliness has become an issue, the idea of the current street cleanliness assessment web portal using mobile edge computing and neural networks, which is a system that helps city municipal authorities to know the cleaning state of the city easily. Training data can be further expanded for better accuracy of the model. Develop a model to detect garbage even in night times or in low light

\section{REFERENCES}

[1] U. Aguileran, O. Pe ${ }^{\sim}$ na, O. Belmonte, and D. L'oopez-de Ipi na, Future Generation Computer Systems, 76, 234-247 (2017)

[2] M. Armbrusit, A. Fox, R. Griffith, A. D. Joseph, R. Katz, A. Konwinski, G. Lee, D. Patterson, A. Rabkoin, I. Stoica et al., Communications of the ACM, 53(4), 50-58 (2010)

[3] C. Badii, P. Beollini, D. Cennoi, A. Difino, P. Nyesi, and M. Paolucci, Future Generation Computer Systems, 75, 14-29 (2017)

[4] C. Balchandani, R. K. Hatwar, P. Makkar, Y. Shah, P. Yeluree, and M. Eirinakiy. (IEEE Third International Conference on Big Data Computing Service and Applications, 2017)

[5] Borozdukhin, O. Dolinina, and V. Pechenkin, (4th IEEE International Colloquium on. IEEE, 2016)

[6]Akila,V.,Sriharshini,K.,Sravani,P.,Sravanthi,D.,Gopi, R.,Sheela,T., International Journal of Online and Biomedical Engineering,17(1),120-128 (2021)
[7] Prasanna Lakshmi K, Reddy CRK. (Int Conf. on Networking and Information Technology,2010)

[8]Hussaini,S.M.,Krishna,G.,Gupta,A.K.,Singh,S.K.,Jou rnal of Manufactoring Processes, 18,151-158(2015)

[9]Sateesh,N.,SampathRao,P.,Ravishankar,D.V.,Satyan arayana,K. ( Materials Today: Proceedings.,2015)

[10] Hussaini, S.M., Singh, S.K., Gupta, A.K., Journal of Materials Research and Technology.,3(1),1724,2014

[11] Dhanalaxmi B, Apparao Naidu, G., Anuradha.K (Procedia Computer Science.,2015) 